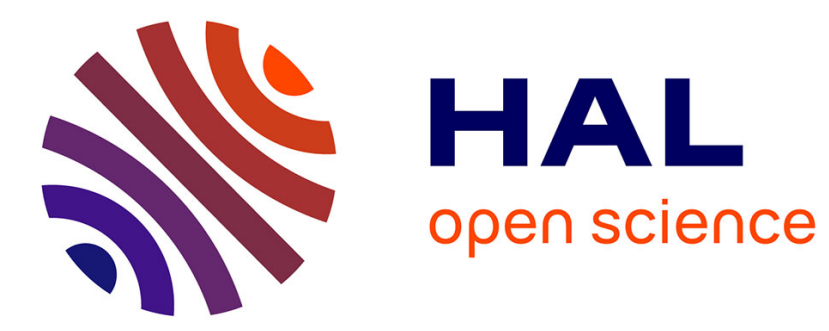

\title{
Transmit frequency optimization for ultrasound constrast agent response
}

Sébastien Ménigot, Anthony Novell, Iulian Voicu, Ayache Bouakaz, Jean-Marc Girault

\section{- To cite this version:}

Sébastien Ménigot, Anthony Novell, Iulian Voicu, Ayache Bouakaz, Jean-Marc Girault. Transmit frequency optimization for ultrasound constrast agent response. Meditech 2009 International Conference on Advancements of Medicine and Health Care through Technology, Sep 2009, Cluj-Napoca, Romania. pp.181-184, 10.1007/978-3-642-04292-8_40 . hal-00612940

\section{HAL Id: hal-00612940 https://hal.science/hal-00612940}

Submitted on 2 Aug 2011

HAL is a multi-disciplinary open access archive for the deposit and dissemination of scientific research documents, whether they are published or not. The documents may come from teaching and research institutions in France or abroad, or from public or private research centers.
L'archive ouverte pluridisciplinaire HAL, est destinée au dépôt et à la diffusion de documents scientifiques de niveau recherche, publiés ou non, émanant des établissements d'enseignement et de recherche français ou étrangers, des laboratoires publics ou privés. 


\title{
Transmit frequency optimization for
}

\section{ultrasound constrast agent response}

\author{
S. Menigot, A. Novell, I. Voicu, A. Bouakaz and J-M. Girault \\ Universite Francois Rabelais de Tours, Inserm U 930, CNRS FRE 2448, Tours, France
}

\begin{abstract}
Introduction: Since the introduction of ultrasound (US) contrast imaging, the imaging systems use a fixed emitting frequency. However it is known that the insonified medium is time-varying and therefore an adapted time-varying excitation is expected. We suggest an adaptive imaging technique which selects the optimal transmit frequency that maximizes the acoustic contrast. Two algorithms have been proposed to find an US excitation for which the frequency was optimal with microbubbles.
\end{abstract}

Methods and Materials: Simulations were carried out for encapsulated microbubbles of 2 microns by considering the modified Rayleigh-Plesset equation for $2 \mathrm{MHz}$ transmit frequency and for various pressure levels $(20 \mathrm{kPa}$ up to $420 \mathrm{kPa})$. In vitro experiments were carried out using a transducer operating at $2 \mathrm{MHz}$ and using a programmable waveform generator. Contrast agent was then injected into a small container filled with water.

Results and discussions: We show through simulations and in vitro experiments that our adaptive imaging technique gives: 1) in case of simulations, a gain of acoustic contrast which can reach $9 \mathrm{~dB}$ compared to the traditional technique without optimization and 2) for in vitro experiments, a gain which can reach $18 \mathrm{~dB}$. There is a non negligible discrepancy between simulations and experiments. These differences are certainly due to the fact that our simulations do not take into account the diffraction and nonlinear propagation effects. Further optimizations are underway.

Keywords - Adaptive imaging, contrast agent, optimization.

\section{INTRODUCTION}

The US contrast imaging domain is in full effervescence. Currently the scientific community of this field seeks US excitations which should make possible the optimization of the acoustic contrast. We tackled the problem in a simple way by proposing an adaptive imaging technique which seeks the emitting frequency that maximizes the acoustic contrast. The use of an adaptive technique is justified by the fact that:
1. during the clinical examination, the insonified medium perfused by the microbubbles is a non-stationary medium (the concentration of bubbles changes...) ;

2. the pressure level is unknown because of the diffraction and attenuation effects which vary from one patient to another ;

3. the size and the distribution of the microbubbles of the contrast agent are not precisely known and can differ from one sample to another.

To overcome these problems, that is to disregard these unknown factors, it seemed more judicious to propose an US excitation whose frequency is selected in an adaptive way using the technique of the gradient ascent or the technique of the golden section search.

\section{METHODS AND MATERIALS}

\section{Methods}

The optimization aims to maximizing the energy of the backscattering signal by iterative method. The principle consists in first measuring the scattered power from the microbubbles, for a sinus wave excitation ; secondly to seek for the optimal transmit frequency by maximizing the scattered power. To avoid the destruction of the microbubbles, the sinus wave train has been modulated with a Gaussian window(fig. 1). Moreover, the length of the pulse has been fixed to the power level was the same for every frequency.

Meditech 2009, International Conference on Advancements of Medicine and Health Care through Technology

The original publication is available at www.springerlink.com

DOI: $10.1007 / 978-3-642-04292-8 \_40$

http://www.springerlink.com/content/r317v02054356831/ 


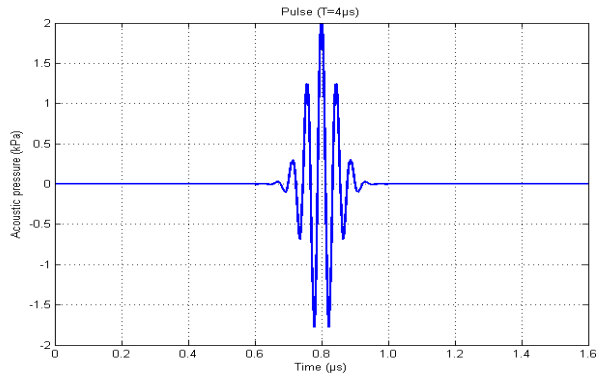

Fig. 1 Emission signal

These optimization methods of scattered signals do not need a priori knowledges of the microbubbles. The single constraint is that power must follow a convex curve on the interval where maximum energy is sought. Two algorithms have been chosen to find the frequency of the truncated sinus incoming signal which maximizes the scattered power:

- golden section search [1]: This approach has been chosen because it does not need to compute the derivative of energy. The first step consists in choosing two frequencies $\left(f_{1}\right.$ and $f_{2}$ ) in the analyzed bandwidth and to compute respectively their energies $\left(E_{1}\right.$ and $E_{2}$ ). From $f_{1}$ and $f_{2}, f_{3}$ and $f_{4}$ and their corresponding energies $E_{3}$ and $E_{4}$ are calculated such as:

$$
\left\{\begin{array}{l}
f_{3}=f_{1}+\rho \cdot \Delta f \\
f_{4}=f_{2}+\rho \cdot \Delta f
\end{array}\right.
$$

where $\Delta f=f_{2}-f_{1}$ and $\rho$ is golden ratio. From the fourth iteration:

a. if $E_{3}>E_{4}, f_{2}=f_{2}, E_{2}=E_{4}, f_{4}=f_{2}$ and $E_{4}=E_{3}$ are assigned. A new $f_{3}$ and its energy are computed.

b. if $E_{4}>E_{3}, f_{1}=f_{3}, E_{1}=E_{3}, f_{3}=f_{4}$ and $E_{3}=E_{4}$ are assigned. A new $f_{4}$ and its energy are computed.

- Gradient ascent [2]: This one maximizes energy with a recurrence relation.

$$
f_{k+1}=f_{k}+\mathbf{\alpha}_{k} \cdot \nabla E\left(f_{k}\right)
$$

Note that the analytic expression of energy according to frequency is unknown. The gradient is estimated with the discrete gradient

$$
E\left(f_{k}\right)=\frac{E_{k}-E_{k-1}}{f_{k}-f_{k-1}}
$$

This ratio is nearly null for small energy in comparison with the frequency. The frequency is modified with a given step $\Delta f$ and $\alpha_{\mathrm{k}}$ has been chosen constant. Note that for every sign change of two successive gradients, $\alpha_{\mathrm{k}}$ is divided by two.

\section{Materials}

\section{Microbubbles}

The ultrasound contrast medium was composed of Sonovue $^{\mathrm{TM}}$ microbubbles [3]. They have a mean size of $2.5 \mu \mathrm{m}$-diameter and $90 \%$ have diameters less than $10 \mu \mathrm{m}$. Their resonance frequency is $2.1 \mathrm{MHz}$ for a $3.2 \mu \mathrm{m}$ bubble [4]. In simulation with Bubblesim [5], the modified Rayleigh-Plesset equation (eq. 4) has been resolved with transducer effects for only one microbubble immersed in water. A microbubble of $2 \mu \mathrm{m}$-radius and $4 \mathrm{~nm}$ shell thickness has been considered.

$$
\begin{gathered}
\ddot{R}(t) \dot{R}(t)+\frac{3}{2} \dot{R}(t)^{2}+\frac{p_{0}+p_{i}(t)-p_{L}(t)}{\rho_{L}} \\
-\frac{R(t)}{\rho_{L} c_{L}} \dot{p}_{L}(t)=0
\end{gathered}
$$

with $R$ instantaneous radius of microbubble and their derivative, $p_{0}$ static pressure, $p_{\mathrm{i}}$ instantaneous acoutic pressure, $p_{\mathrm{L}}$ liquid pressure on the surface of the microbubbles, $\rho_{0}$ liquid density and $c_{\mathrm{L}}$ velocity of sound in the liquid.

Their backscattered energy has been computed with a mean energy on ten received signals to cancel out the motion of microbubbles.

\section{i) Acoustical Measurements}

Each method was compiled using Matlab ${ }^{\circledR}$ (Mathworks, Natick, MA) and the resulting excitation signal was transmitted through a GPIB port (National Instruments, Austin, TX) to an arbitrary function generator (33220A, Agilent, Palo Alto, CA). The signal was then

Meditech 2009, International Conference on Advancements of Medicine and Health Care through Technology The original publication is available at www.springerlink.com DOI: 10.1007/978-3-642-04292-8_40 
amplified using a power amplifier (Amplifier Research 150A100B, Souderton, PA) and transmitted to a $1.9 \mathrm{MHz}$ PZT single element (Sofranel, Sartrouville, France) focused at 55 $\mathrm{mm}$ and with a bandwidth of $80 \%$ (fig. 3 ).

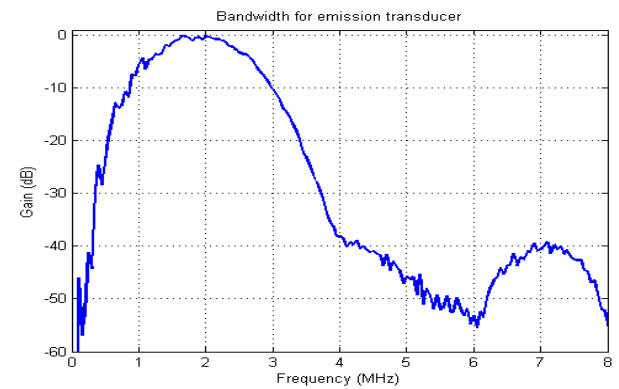

Fig. 2 Bandwidth of emission transducer

Responses of a 1/2000 diluted solution of Sonovue ${ }^{\mathrm{TM}}$ microbubbles (Bracco Research, Geneva, Switzerland) were measured by a 3.5 $\mathrm{MHz}$ PZT single element transducer with a bandwidth of $63 \%$ (fig. 3) also focused at 55 $\mathrm{mm}$ and used in receive mode.

Both transducers were placed perpendicularly to avoid direct transmission (fig. 4).

Measured echoes were amplified by $30 \mathrm{~dB}$ (Panametrics, Sofranel, Sartrouville, France) and then visualized on a digital oscilloscope (Tektronix, Beaverton, OR). Signals were finally transferred to a personal computer through a GPIB port for further analysis. The effects of transducers have been compensated with their bandwidth by an amplitude coefficient.

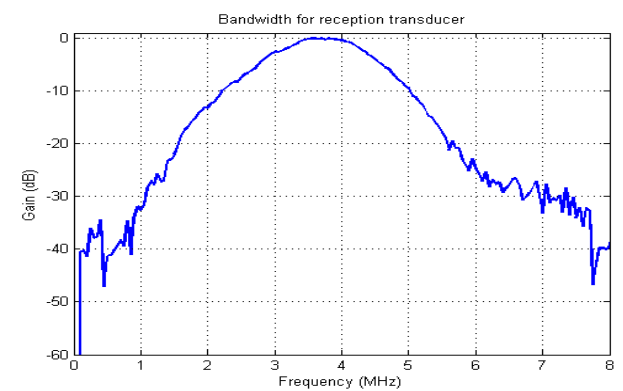

Fig. 3 Bandwidth of reception transducer

To compare the adaptive technique to the traditional technique, the central frequency of emission transducers was a reference frequency.

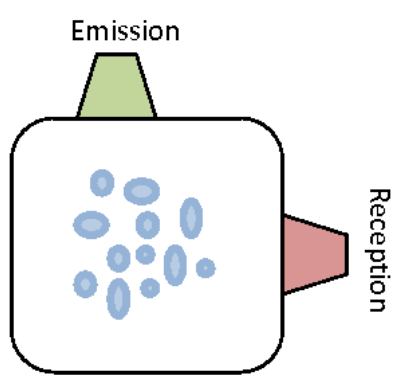

Fig. 4 System

RESULTS

\section{Simulations}

To know the evolution energy according to the frequency, a microbubble has been stimulated during $4 \mu$ s and the backscattering signal has been computed for different pressures.

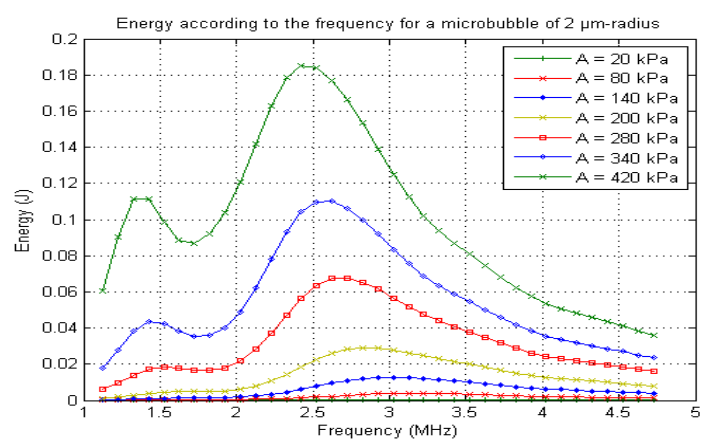

Fig. 5 Energy according to frequency for a microbubble of $2 \mu$ m radius

The frequency which allows obtaining a maximum energy varies with the pressure: for a high pressure, the frequency is lower (fig 5). From the appearance of energy, the initial frequencies for the algorithms have been chosen to find global maximum.

The golden section search is applied with three signals of $140 \mathrm{kPa}, 280 \mathrm{kPa}$ and $480 \mathrm{kPa}$ maximal pressure.

Meditech 2009, International Conference on Advancements of Medicine and Health Care through Technology The original publication is available at www.springerlink.com DOI: $10.1007 / 978-3-642-04292-8 \_40$

http://www.springerlink.com/content/r317v02054356831/ 

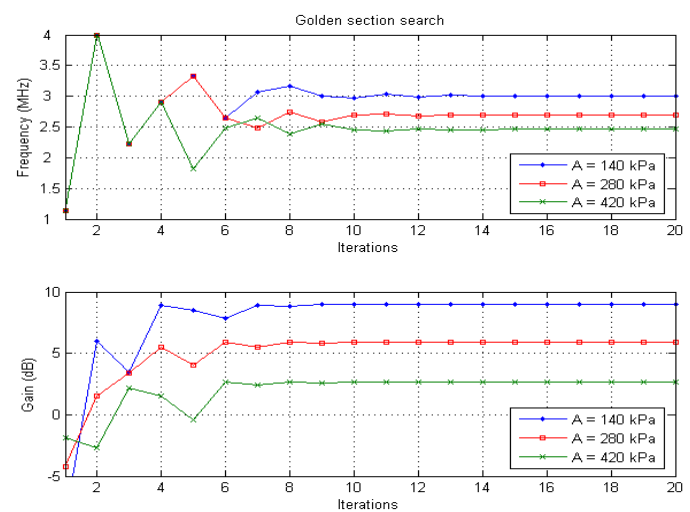

Fig. 6 Golden section search for a microbubble of $2 \mu \mathrm{m}$ radius

The optimal frequencies permit to have a positive gain which reach $8.95 \mathrm{~dB}$ for a pressure of $140 \mathrm{kPa}$ (fig. 6). They are stable after ten iterations. For each pressure, the optimal frequency is different $(2.99 \mathrm{MHz}, 2.69 \mathrm{MHz}$ and $2.46 \mathrm{MHz}$ respectively for $140 \mathrm{kPa}, 280 \mathrm{kPa}$ and $480 \mathrm{kPa}$ ), which the energy according to the frequency showed.

The optimization has been done again with the same signal amplitude.
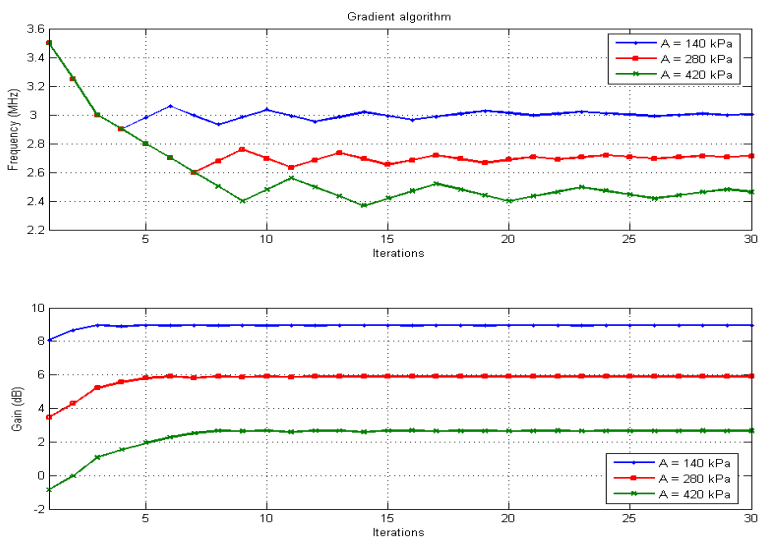

Fig. 7 Gradient ascent for a microbubble of $2 \mu \mathrm{m}$ radius

The gradient ascent has found the same optimal frequencies (fig 7).

\section{Experiments}

To compute the optimal frequency, a cloud of microbubbles has been stimulated during $10 \mu \mathrm{s}$ to avoid an impulse stimulation. Three pressures $(140,280$ and $420 \mathrm{kPa})$ have been applied.
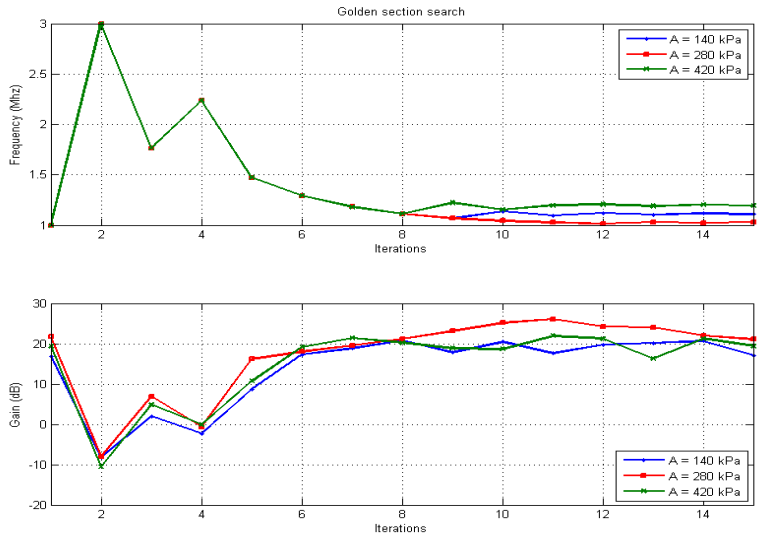

Fig. 8 Experiment with golden section search

For the golden section search, the optimal frequency has been found around $1.1 \mathrm{MHz}$ with a gain which can reach $18 \mathrm{~dB}$ (fig. 8).
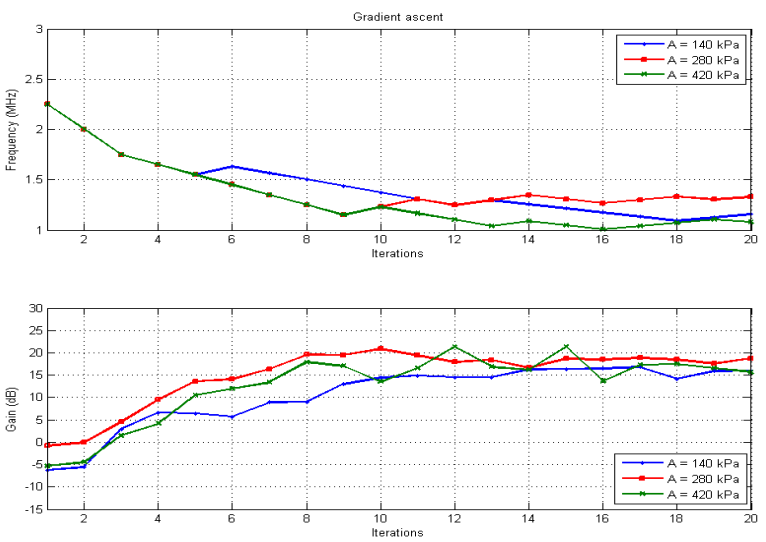

Fig. 9 Experiment with gradient ascent

For the gradient ascent, the optimal frequency fluctuated between $1.3 \mathrm{MHz}$ for $280 \mathrm{kPa}$ and 1.1 MHz for $420 \mathrm{kPa}$ (fig. 9). The gains are similar compared with the other algorithm, it is around $18 \mathrm{~dB}$.

\section{Discussions}

These adaptive imaging techniques have allowed to:

Meditech 2009, International Conference on Advancements of Medicine and Health Care through Technology The original publication is available at www.springerlink.com DOI: $10.1007 / 978-3-642-04292-8 \_40$

http://www.springerlink.com/content/r317v02054356831/ 
- in case of simulations, a gain which can reach $9 \mathrm{~dB}$ compared to the traditional technique,

- for in vitro experiments, a gain which can reach $18 \mathrm{~dB}$.

According to the pressure applied to microbubbles, the optimal frequency changes. After around ten iterations, the optimal frequency has been found and the energy of the microbubbles is stable.

The experimental results are not exactly identical because of the non-stationarity of microbubbles. To cancel their motion, we have repeated the experiment. But a high number of repetition and a high value of iterations number could destroy the microbubbles and thus the energy could decrease. A trade-off must be found to avoid the destruction of the microbubbles.

These optimization could optimize the harmonic frequency of the microbubbles with our couple of transducers. The reception transducer has a bandwidth which corresponds with this frequency.

\section{CONCLUSIONS}

The optimization allows to have a not- inconsiderable gain for energy of backscattering signal. These methods allow to find the optimal frequency adapted to microbubbles.

We can carry on with complicating the optimization, first of all, with finding the optimal frequency and the optimal amplitude simultaneously. Then we will try to distort a sinus curve in order to be close to the form described by Szeri [6].

\section{REFERENCES}

1. W. H. Press, B. P. Flannery, S. A. Teukolsky, and W. T. Vetterling, Numerical Recipes in Fortran, 2nd ed. Cambridge University Press, January 1992.

2. F. Michaut, Méthodes adaptatives pour le signal. Paris: Hermes, 1992.

3. C. Greis, "Technology overview: SonoVue (Bracco, Milan)," EurRadiol Suppl, vol. 14, no. 8, pp. 11-15, October 2004.

4. Ir. S.M. van der Meer, Dr. M. Versluis, Prof.Dr. D. Lohse, C.T. Chin, A. Bouakaz, and Prof.Dr. N. de Jong, "The resonance frequency of SonoVue (tm) as observed by high-speed optical imaging," in IEEE Ultrasonics Symposium, 2004, vol. 1. IEEE, 2004, pp. 343-345.

5. L. Hoff, Acoustic Characterization of Contrast Agents for Medical

Ultrasound Imaging. Boston: Kluwer Academic, 2001.

6. A. Reddy and A. Szeri, "Optimal pulse-inversion imaging for microsphere contrast agents," Ultrasound in Med. \& Biol., vol. 24, no. 4, pp. 483-494, 2002. 